ISSN 00156043

NOTAS

\title{
El desarrollo económico de Paraguay: fracaso de su modelo de financiación'
}

\section{Juan Vicente Fruet Cardozo²}

Palabras claves: Paraguay, Financiación para el desarrollo, Carreteras, Salud y Saneamiento, Educación, Vivienda.

Key words: Paraguay, Financial aid for Development, Highways, Health and Sanitation, Education, Housing

\section{Introducción}

Entre los años 1950 y 2000, Paraguay recibió de los principales organismos financieros de la región latinoamericana, el Banco Interamericano de Desarrollo (BID), y en menor medida, del Banco Internacional de Reconstrucción y Fomento, más conocido como Banco Mundial (BM), en concepto de financiación para su desarrollo, la suma de 2.037.281.391,36 dólares americanos (en adelante, "dólares"). Dicho importe, expresado en dólares del año 2000

\footnotetext{
1 Este trabajo es un resumen del contenido de la tesis doctoral del autor, defendida en la Facultad de Ciencias Económicas y Empresariales (ETEA), adscrita a la Universidad de Córdoba, en julio de 2006. Aprobado para publicación el 13 de marzo de 2008. Con posterioridad tuvieron lugar las elecciones en que salió vencedor Fernando Lugo a quien se alude en otro lugar de este texto.

${ }^{2}$ Profesor del Departamento de Economía, Sociología y Política Agrarias, Área de Economía Financiera y Contabilidad de la Facultad de Derecho y Ciencias Económicas y Empresariales y de la Facultad de Ciencias del Trabajo, de la Universidad de Córdoba. Consultor Financiero Internacional en proyectos de desarrollo del sector público y privado.
} 
para homogeneizar los datos, representaba la suma de 3.280.055.412,00 dólares ${ }^{3}$.

\begin{tabular}{|l|r|r|r|r|r|}
\hline \multirow{2}{*}{ Tabla N $N^{\circ}$ 1. Financiaciones aprobadas para Paraguay del BM y del BID. } \\
Período $\mathbf{1 9 5 0}$ a $\mathbf{2 0 0 0}$
\end{tabular}

Fuente: Banco Internacional de Reconstrucción y Fomento (BIRF) o Banco Mundial y Banco Interamericano de Desarrollo (BID). Gerencias Regionales. Washington, DC. EE. UU.

La Tabla $N^{\circ} 1$ presenta el consolidado de las financiaciones aprobadas por ambos organismos.

Asimismo, resulta importante recordar que junto a las instituciones mencionadas, también otros organismos multilaterales y bilaterales han prestado su financiamiento y cooperación al país. Dichos organismos son: el Japan Bank for Internacional Cooperation (JBIC) y el Overseas Economic Cooperation Fund of Japan (OECF), ambos del Gobierno de Japón; el Gobierno de Taiwán; el Fondo Monetario Internacional (FMI); el Deutsche Gesellchaft für Tecnische Zusammenarbeit (GTZ); el Kreditanstalt für Wiederanfban (KFW); el Fondo Financiero para el Desarrollo de la Cuenca del Río de la Plata (FONPLATA) y la Corporación Andina de Fomento (CAF). De igual manera, fueron importantes las financiaciones de gobiernos de los países de la Unión Europea (Alemania, España, Francia, Bélgica, Holanda y Gran Bretaña), así como de Estados Unidos de Norteamérica, fundamentalmente a través de su Agencia Internacional de Desarrollo (AID).

Por lo tanto, la cantidad destinada al desarrollo de Paraguay durante los últimos cincuenta años del siglo XX, fue bastante superior a las cifras indicadas en el primer párrafo de este artículo.

Los comentarios expuestos con anterioridad quieren dejar entrever que los recursos financieros desembolsados, tuvieron que haber sido suficientes para iniciar y apuntalar el desarrollo de un pequeño país como Paraguay, siempre que los mis-

\footnotetext{
${ }^{3}$ Importe equivalente en la actualidad a unos 2.150.000.000 euros.
} 
mos hubiesen sido invertidos adecuadamente. No obstante, a la luz de la precaria situación económica y social del país, ly, por qué no decirlo, de toda América Latina y el Caribe ${ }^{4}$ ), se puede afirmar con rotundidad que no ha ocurrido así. Por el contrario, salta a la vista el sostenido deterioro del tejido económico-social sufrido por Paraguay en la segunda mitad del siglo XX, acrecentado durante la década de los noventa, y que se manifiesta plausiblemente hasta nuestros días.

En términos específicos, las raíces del problema son, por un lado, (i) la corrupta y delirante administración del país encabezada por la autoritaria figura del General Alfredo Stroessner Matiauda (1954-1989); (ii) las débiles y también corruptas administraciones democráticas iniciadas a partir de 1989 hasta nuestros días; y, (iii) la débil gestión de los principales organismos multilaterales de desarrollo en lo que concierne al diseño, análisis, ejecución, "inspección y vigilancia", así como la evaluación ex post de los centenares de programas financiados a través de multimillonarios préstamos concedidos al país 5 .

Al respecto, después de contrastar cada una de las financiaciones desembolsadas, frente a los pobres resultados logrados, la investigación ha propuesto la adopción de otros modelos yotras fuentes alternativas de financiación para reimpulsar el desarrollo paraguayo, los cuales, por motivos de espacio, no forman parte de este artículo.

\section{Datos básicos de Paraguay}

Paraguay, fue descubierto en el año 1524 por el español Alejo García, y su capital, Nuestra Señora Santa María de la Asunción (más conocida como Asunción), fundada en 1537, por el capitán Juan de Salazar y Espinoza. Se independizó de la Corona española el 14 de Mayo de 1811, y en los siguientes días, los próceres de la independencia la transformaron en República unitaria, tal como permanece hasta nuestros días.

\footnotetext{
${ }^{4}$ Chile -aunque todavía persistan problemas de repartición de la riqueza- configura la excepción en América Latina. Así como es evidente el deterioro generalizado del conjunto de los países latinoamericanos, resulta innegable el nivel de desarrollo alcanzado por la economía chilena, iniciado bajo el régimen del General Augusto Pinochet Ugarte, fallecido a finales de 2006. Su política económica se basó en la economía de mercado.

${ }^{5}$ La constatación real de estos hechos a través de experiencias profesionales vividas directamente en programas de desarrollo en Paraguay, así como en otros países de América Latina y del Caribe, han constituido el leitmotiv para analizar durante años -y seguir haciéndolo- el delicado y espinoso, pero, trascendental tema de la financiación para el desarrollo.
} 
Tiene una superficie de $406.752 \mathrm{~km}^{2}$, no posee costas marítimas, y se divide en dos regiones; la región Occidental o Chaco, más bien seca, y la región Oriental, fértil y con abundantes ríos ${ }^{6}$. Esta región se halla sobre el denominado "Acuífero Guarani", que constituye uno de las reservas de agua subterránea dulce más grande del mundo 7 .

El país, por su naturaleza, es agrícola-ganadero. Posee una población de aproximadamente 5.800.000 habitantes, siendo su densidad demográfica de apenas unos 14,5 habitantes por $\mathrm{Km}^{2}$. Su moneda es el guaraní, cuya paridad actual con el euro es de aproximadamente $1 €=7.000 \mathrm{G}$. Sus lenguas oficiales son el castellano y el guaraní 8 .

\section{Breve reseña de la historia político-económica (1950-2000)}

La historia económica y política de Paraguay correspondiente a la segunda parte del siglo XX, puede dividirse en tres etapas:

1. 1950 a 1954: años de agudo estancamiento económico y de anarquía política, que se había iniciado unos 80 años atrás?

\footnotetext{
${ }^{6}$ Durante la década de los cincuenta e inicios de los sesenta, Paraguay, aunque escaso en población y en desarrollo industrial, era, sin embargo, muy rico en recursos naturales. La Región Oriental tenía un $70 \%$ de cobertura boscosa, se hallaba regada por innumerables cursos de agua, el suelo era fértil y el clima estable. Constituía una reserva potencial de alimento para millones de personas. Hoy en día, continúa siendo pobre en desarrollo industrial, pero, lamentablemente, ahora lo es en muchos otros aspectos. Como consecuencia de una tala indiscriminada de sus bosques, se perdió la cobertura vegetal, y con ello, mucha de su fertilidad; de igual manera, más de la mitad de los numerosos cursos de agua desaparecieron; sus ríos y arroyos se hallan contaminados o sus caudales se redujeron significativamente.
}

${ }^{7}$ El "Acuífero Guaraní" se halla en el subsuelo de un área de alrededor de 1.190.000 kilómetros cuadrados (superficie mayor que las de España, Francia y Portugal juntas). También se lo denomina el "Acuífero Gigante del Mercosur", porque abarca subsuelos de Brasil, Argentina, Paraguay y Uruguay.

${ }^{8}$ El guaraní es la lengua de la raza indígena que poblaba todo lo que es el Paraguay actual, el norte argentino, parte del oeste brasileño y el sur boliviano. En la actualidad, es el único caso en el continente en el que un idioma indígena es hablado por todos sus habitantes.

9 En el siglo XIX, desde 1864 hasta 1870, Paraguay sostuvo un conflicto bélico, conocido como "Guerra de la Triple Alianza". Durante esos años, se enfrentó con Brasil, Argentina y Uruguay. Los nefastos resultados fueron: (i) la muerte de un $60 \%$ de sus habitantes (1.200.000 personas), donde 
2. 1954 a 1989: un largo período de 35 años de régimen dictatorial centralizado en la figura del General Alfredo Stroessner Matiauda. Durante el mismo se llevaron a cabo algunas importantes obras de infraestructura, pero, sus resultados arrojaron un pronunciado déficit social y una herencia de corrupción sin precedentes en el país.

3. 1989 a 2000 (hasta la actualidad): inicio de una serie de gobiernos democráticos, los cuales demostraron una extrema inoperancia en la administración del país.

Desde 1947, Paraguay está gobernado por un partido político denominado Asociación Nacional Republicana, conocida como Partido Colorado. El 20 de abril de 2008 se realizarán las elecciones presidenciales, y, por primera vez, después de más de seis décadas, el Partido Colorado corre seriamente el peligro de perder las elecciones.

\section{Financiaciones para el desarrollo recibidas entre I 950 a 2000}

Los sectores analizados han sido: Carreteras, Salud y Saneamiento, Educación y Vivienda. A continuación se presenta la situación de los principales indicadores de dichos sectores, entre 1950 2000, así como las respectivas financiaciones:

\section{I. Carreteras}

\begin{tabular}{|l|c|c|c|}
\hline \multicolumn{3}{|c|}{ Tabla N $N^{\circ}$ 2. Sector Carreteras } \\
\hline \multirow{2}{*}{ Indicadores } & \multicolumn{2}{|c|}{ Años y datos } & \multirow{2}{*}{$\begin{array}{c}\text { Financiaciones } \\
\text { externas } \\
1954-2000\end{array}$} \\
\cline { 2 - 3 } & 1950 & 2000 & \\
\hline Inventario caminero en kilómetros & 1.138 & 26.904 & \\
\hline Kilómetros de rutas asfaltadas & 87 & 2.497 & \multirow{2}{*}{ USD 707.345.000 } \\
\hline $\begin{array}{l}\text { \% de kilómetros de rutas asfaltadas sobre } \\
\text { el inventario de obras viales }\end{array}$ & $7,6 \%$ & $9,3 \%$ & \\
\hline Kilómetros de caminos terraplenados & 1.051 & 24.407 & \\
\hline
\end{tabular}

Fuente: Ministerio de Obras Públicas y Comunicaciones (MOPC). Dirección General de Vialidad. Asunción, Paraguay.

la población masculina fue diezmada; (ii) la pérdida de $200.000 \mathrm{~km}^{2}$ de territorio, anexados en su mayor parte a Brasil; y, (iii) el colapso de la ordenada y próspera economía paraguaya. Las secuelas de esa hecatombe subsisten hasta nuestros días. 
Desde el punto de vista cuantitativo, las mejoras observadas son importantes; sin embargo, la infraestructura vial paraguaya al año 2000, a pesar de los 707.345.000 de dólares prestados, no se adecua a los requerimientos del país, ni de la región. Si bien se pavimentaron 2.410 kilómetros (87 kilómetros, en 1954, y 2.497 kilómetros, en 2000) en 46 años, un faltante de 3.518 kilómetros que hemos detectado en nuestro estudio ${ }^{10}$, así como las numerosas obras realizadas sin haberse cumplido las especificaciones técnicas requeridas, dan lugar al enorme déficit actual de miles de kilómetros de carreteras ${ }^{11}$.

\subsection{Salud y Saneamiento}

\begin{tabular}{|c|c|c|c|}
\hline \multicolumn{4}{|c|}{ Tabla Nº 3. Sector Salud y Saneamiento } \\
\hline \multirow{2}{*}{ Indicadores } & \multicolumn{2}{|c|}{ Años y datos } & \multirow{2}{*}{$\begin{array}{c}\text { Financiaciones } \\
\text { externas } \\
1954-2000\end{array}$} \\
\hline & 1954 & 2000 & \\
\hline \% población sin seguro médico & N.D. & $81 \%$ & \multirow{5}{*}{$\begin{array}{c}\text { USD } \\
418.213 .000\end{array}$} \\
\hline Tasa de mortalidad infantil & $90 \%$ & $27 \% 0^{1}$ & \\
\hline Tasa de mortalidad materna & N.D. & $160 \% \%^{2}$ & \\
\hline $\begin{array}{l}\% \text { de población sin acceso a agua } \\
\text { potable }\end{array}$ & N.D. & $41 \%$ & \\
\hline $\begin{array}{l}\% \text { de población sin acceso a saneamiento } \\
\text { básico }\end{array}$ & N.D. & $90 \%{ }^{3}$ & \\
\hline
\end{tabular}

\footnotetext{
${ }^{1}$ Tanto por $\mathrm{mil}=\%$.

${ }^{2}$ Tanto por cien $\mathrm{mil}=\%$ oo.

${ }^{3} 30 \%$ de la población utiliza sistemas no sanitarios, y el 60\%, "soluciona" individualmente, a través de fosas sépticas, sin mayores controles.
}

Fuentes: Ministerio de Salud Pública y Bienestar Social, (MSP y BS), Asunción, y Dirección General de Estadísticas, Encuestas y Censos (DGEE y C), Fernando de la Mora, Paraguay.

${ }^{10}$ Este tema es analizado en detalle en el Capítulo 4 de la tesis doctoral.

1 En el sector vial, para que Paraguay pueda ponerse a la altura de los demás países que integran el denominado Mercado Común del Sur (MERCOSUR), debe tener asfaltados 8.000 kilómetros de carreteras. 
Al igual que el sector Carreteras, los préstamos externos que alcanzaron 418.213.000 de dólares - de los cuales, la mayor parte fueron obtenidos durante la década de los noventa- no tuvieron impactos relevantes en la población, porque la situación de la salud y el saneamiento básico es claramente mejorable. Esto se constata en realidades tales como: el $81 \%$ de la población no posee seguro médico-hospitalario, y, hacia fines de los noventa, las tasas de mortalidad infantil y materna, empezaron de nuevo a incrementarse hacia niveles preocupantes, tal como se indica en el cuadro. Asimismo, las tasas de la población carente de servicios básicos de saneamiento-agua potable y alcantarillado sanitario, $41 \%$ y $90 \%$, respectivamente- son alarmantes.

\subsection{Educación}

\begin{tabular}{|l|c|c|c|}
\hline \multicolumn{2}{|c|}{ Tabla $N^{\circ}$ 4. Sector Educación } \\
\cline { 1 - 3 } Indicadores & \multicolumn{2}{|c|}{ Años y datos } & \multirow{2}{*}{$\begin{array}{c}\text { Financiaciones } \\
\text { exfernas } \\
1960-2000\end{array}$} \\
\cline { 2 - 3 } & 1960 & 2000 & \\
\hline Tasa de absorción escolar (1960) & $44,3 \%$ & $68,2 \%$ & \\
\hline Tasa de retención escolar (1970) & $25,5 \%$ & $48,0 \%$ & \\
\hline Tasa de analfabetismo (1962) & $27,6 \%$ & $6,2 \%$ & \multirow{2}{*}{ USD 193.567 .000} \\
\hline$N^{\circ}$ de escuelas primarias (1960) & 2.271 & 7.958 & \\
\hline$N^{\circ}$ de alumnos por escuela (1960) & 134,5 & 158,9 & \\
\cline { 1 - 3 }$N^{\circ}$ de alumnos por maestro (1960) & 28,5 & 19,4 & \\
\hline
\end{tabular}

Fuentes: Ministerio de Educación y Cultura (MEC), Asunción, y Dirección General de Estadísticas, Encuestas y Censos (DGEE y C), Fernando de la Mora, Paraguay.

A excepción del progreso observado en la tasa de analfabetismo, las mejoras observadas en 40 años, pertenecen también a aspectos meramente cuantitativos, porque los problemas actuales relacionados con la calidad educativa, son cada vez más acuciantes. En este sentido, el reciente fracaso del Programa de Reforma Educativa, iniciada en 1994, ha dejado como principal herencia, el aumento del saldo de la deuda externa.

A modo de ejemplo, si sólo nos concentráramos en el grave e incomprensible error sobre la estimación de la tasa de retención escolar (permanencia en el puesto 
escolar) para el 2000'2, el mismo resulta suficiente para inferir que el análisis de factibilidad fue elaborado de manera técnicamente irresponsable, muy alejado de la realidad paraguaya.

El escenario actual en que se desenvuelve la población escolar del país se halla constituido por centenares de miles de niños, adolescentes y jóvenes, sin las mínimas comodidades en las aulas -muchas escuelas primarias dan clases bajo árboles, incluso en Asunción- mobiliario desvencijado, carentes de textos básicos y útiles escolares; bibliotecas sin libros, laboratorios desprovistos de materiales, y unos 9.000 maestros trabajando ad honorem.

Resulta paradójico que el actual Presidente del país, Nicanor Duarte Frutos, haya sido Ministro de Educación y Cultura dos períodos consecutivos; y la actual candidata presidencial por el Partido Colorado, Blanca Ovelar, también fue Ministra de dicha cartera.

\subsection{Vivienda}

\begin{tabular}{|c|c|c|c|}
\hline \multicolumn{4}{|c|}{ Tabla Nº 5. Sector Vivienda } \\
\hline \multirow{2}{*}{ Indicadores } & \multicolumn{2}{|c|}{ Años y datos } & \multirow{2}{*}{$\begin{array}{c}\text { Financiaciones } \\
\text { externas } \\
1960-2000\end{array}$} \\
\hline & 1950 & 2000 & \\
\hline$N^{\circ}$ de viviendas & 244.798 & 1.175 .573 & \multirow{5}{*}{ USD 57.400 .000} \\
\hline $\mathrm{N}^{\circ}$ de viviendas urbanas & 88.616 & 699.845 & \\
\hline $\mathrm{N}^{\circ}$ de viviendas rurales & 156.182 & 475.728 & \\
\hline Déficit habitacional & N.D. & 453.509 & \\
\hline $\mathrm{N}^{\circ}$ de personas por vivienda & 5,4 & 4,7 & \\
\hline
\end{tabular}

Fuente: Dirección General de Estadísticas, Encuestas y Censos (DGEE y C). Fernando de la Mora, Paraguay.

El déficit habitacional del país, en el año 2000 , era de 453.509 viviendas, que afectaba a unas 2.132 .000 personas (36,8\% de la población del país). Pero, no existe una política sectorial en materia de vivienda. Por tanto, este enorme segmento poblacional se halla abandonado a su suerte.

12 Los estudios de factibilidad del Programa de Reforma Educativa, realizados entre 1993 y 1994 , estimaron que la tasa de retención escolar en el año 2000, iba a alcanzar el 100\%. Cuando se realizaron dichos estudios dicho ratio estaba en $52 \%$, y en el 2000 ... jbajó al 48\%! 
Nuestro estudio presenta una propuesta de financiación para la mitad de la población afectada por la carencia habitacional -que, en base a una clasificación por nivel salarial anual- denominamos "primer grupo" y "segundo grupo"Representa 1.000.000 de personas.

\begin{tabular}{|c|c|c|c|}
\hline \multicolumn{3}{|c|}{ Tabla $\mathrm{N}^{\circ}$ 6. Sector Vivienda. Clasificación de la población involucrada } \\
\hline Grupo y nivel salarial anual & Nivel & $\begin{array}{c}\mathbf{N}^{\circ} \text { de } \\
\text { personas }\end{array}$ & $\begin{array}{c}\mathbf{N}^{\circ} \text { de viviendas } \\
\text { necesarias }\end{array}$ \\
\hline $1^{\circ}:(2.160 \$)$ & Medio bajo & 500.000 & 106.383 \\
\hline $2^{\circ}:$ (de 1.620 a 2.136 \$) & Bajo & 500.000 & 106.383 \\
\hline $3^{\circ}$ : (menor que 626 \$) & Muy bajo & 1.131 .492 & 240.743 \\
\hline Total & & $\mathbf{2 . 1 3 1 . 4 9 2}$ & $\mathbf{4 5 3 . 5 0 9}$ \\
\hline
\end{tabular}

Sin embargo, para el que llamamos "tercer grupo", en el cual se encuentra el 20\% de la población del país que vive en la extrema pobreza-unas 1.131 .492 personas que necesitan unas 240.743 viviendas- no existen posibilidades de adquirir una vivienda mínima y digna nunca, porque:

(i) Las debilitadas finanzas públicas del país, hoy, y con seguridad, en los próximos años, no podrán atender esta necesidad.

(ii) Para los organismos multilaterales de desarrollo, el sector vivienda no configura una prioridad en sus agendas, hecho que puede corroborarse al observar que, en 50 años, sólo se destinaron 57.400 .000 de dólares $(4,2 \%$ del total de financiaciones para los cuatro sectores analizados, 1.375.525.000 de dólares). Además, las contadas operaciones han sido un completo fracaso ${ }^{13}$.

(iii) Por otra parte, las fuerzas del mercado de la vivienda impulsadas por el sector privado, no llegan hasta estos niveles de la población.

\subsection{Resumen}

Este es el panorama desolador que presenta el país después de cinco décadas de haber recibido multimillonarias financiaciones externas para el desarrollo de los

\footnotetext{
${ }^{13} \mathrm{~A}$ modo de ejemplo, cabe señalar que el programa BID/CONAVI, financiado con recursos de los préstamos del Banco Interamericano de Desarrollo, 683/OC-PR y 884/SF-PR, resultó un escándalo, donde la corrupción de funcionarios del BID, junto con sus pares de la administración paraguaya, fueron publicados con lujo de detalles en la prensa.
} 
cuatro sectores mencionados. La tabla siguiente resume dichas financiaciones:

\begin{tabular}{|c|c|}
\hline \multicolumn{2}{|c|}{$\begin{array}{l}\text { Tabla N }{ }^{\circ} \text { 7. Financiaciones externas totales de los cuatro } \\
\text { sectores analizados }\end{array}$} \\
\hline Sector & USD \\
\hline - Carreteras & 707.345 .000 \\
\hline - Salud y Saneamiento & 418.213 .000 \\
\hline - Educación & 193.567.000 \\
\hline - Vivienda & 57.400 .000 \\
\hline TOTAL & USD $1.376 .525 .000^{1}$ \\
\hline
\end{tabular}

' Tal como lo indica el título de la Tabla $\mathrm{N}^{\circ} 7$, este importe incluye el total de la financiación externa para los cuatro sectores analizados. Por tanto, no coincide con la cifra de 2.037.281.391,36, consignada en la Tabla $N^{\circ} 1$, que es el total de la financiación del BID y del Banco Mundial desembolsado para todos los sectores económicos.

De todo esto, lo que sí persiste es el saldo de la deuda externa paraguaya, asunto del cual nos ocupamos a continuación.

\section{La deuda externa de Paraguay}

\section{I. Consideraciones generales}

Antes de comentar este tema, recordemos que existen numerosísimos estudios y artículos sobre la deuda externa, escritos por profesionales de todo tipo de disciplinas. Y, prácticamente, todos presentan a la deuda casi como demoníaca.

No obstante, una deuda -sea del sector público o privado- no es mala, ni buena, en sí misma. Es simplemente una modalidad de obtener recursos de terceros, debido a la dificultad de utilizar fondos propios, o por la conveniencia financiera basada en la técnica denominada "leverage", en el caso microeconómico, o, por la insuficiencia del ahorro interno, en el caso macroeconómico.

Una vez presentada la solicitud y efectuados los trámites de rigor, se aprueban las operaciones. Así, los fondos son prestados para llevar a cabo proyectos, y, tanto 
la administración pública -o la empresa privada de ser el caso- y la institución prestamista son responsables conjuntamente de la aplicación adecuada de dichos fondos ${ }^{14}$. Por tanto, es clave la correcta utilización de los recursos para que los proyectos -públicos o privados- funcionen y el repago sea factible. Esto sería lo correcto. Por el contrario, el uso inapropiado y/o la dilapidación de los fondos -la corrupción-, es el verdadero problema.

\subsection{Evolución y saldo de la deuda externa paraguaya}

La evolución de la deuda externa paraguaya desde 1950 a 2000 se observa a continuación:

\section{Tabla $N^{\circ} 8$. Evolución de la deuda externa paraguaya 1950-2000}

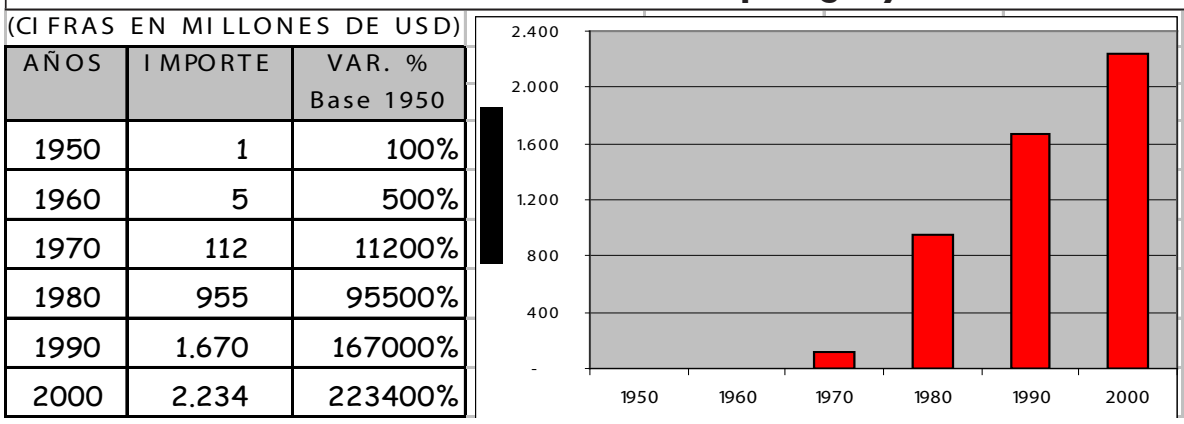

Fuente: Banco Central del Paraguay. Departamento de Estudios Económicos. Asunción.

El crecimiento ha sido espectacular: de un nivel de endeudamiento inexistente, en 1950, 50 años después se fue incrementando hasta 2.234.000.000 de dólares, que configuraba el saldo al 31 de diciembre de 2000. El incremento representa un ¡ $223.400 \%$ ! El festival de endeudamiento empezó en los años ochenta, y en los noventa, con el advenimiento de la democracia, alcanzó niveles técnicamente inaceptables.

Sin embargo, lo grave del caso es que este saldo no incluye la deuda contraída para la construcción de las dos presas binacionales: Itaipú, con Brasil, y Yacyretá, con Argentina. El saldo de la deuda -sólo de Itaipú- asciende

\footnotetext{
${ }^{14}$ En el caso de los préstamos internacionales, esta corresponsabilidad se plasma taxativamente en una cláusula contractual.
} 
actualmente a la colosal suma de unos 18.000.000.000 de dólares. Esta cifra no se halla contabilizada como deuda externa por no contar con el aval del Gobierno de Paraguay ${ }^{15}$. ¡Pero, los paraguayos pagan anualmente el servicio de la misma!

\subsection{Impacto de la deuda externa en Paraguay}

¿Para qué ha servido esta deuda? En base a lo expuesto en el apartado 4, repasemos rápidamente los principales problemas de los sectores analizados:

5.3.1. Carreteras: faltante de 3.518 kilómetros de carreteras pavimentadas, gran parte del inventario caminero no reúne las especificaciones técnicas básicas, tales como anchura de las rutas, banquinas, señalizaciones horizontales ni verticales, entre otros numerosos aspectos.

5.3.2. Salud y saneamiento básico: $81 \%$ de la población sin cobertura médicohospitalaria, y aunque lo tuviera, si un paraguayo contrajese alguna enfermedad medianamente grave, la probabilidad de que muera es alta (si recurre al sector privado, los costes de la sanidad son similares a los del primer mundo); las tasas de mortalidad materno-infantil, en los últimos años se volvieron a disparar hacia niveles del tercer mundo.

5.3.3. Educación: apenas $48 \%$ de retención escolar, tanto en el ciclo primario, como en el secundario; escuelas primarias en estado deplorable; miles de maestros trabajando ad honorem; bibliotecas sin libros, etc.

5.3.4. Vivienda: $36,8 \%$ de la población (2.132.000 personas) con déficit habitacional, de los cuales 1.132 .000 no tienen posibilidad de tener una vivienda mínima digna nunca.

Frente a lo expuesto, lo cual resulta evidente con sólo observar hoy las calles, las plazas y las esquinas con semáforos de las ciudades y pueblos del país, y compararlo con la situación de hace 30 años, sin temor a equívocos, podemos afirmar que el modelo de financiación para el desarrollo de Paraguay ha fracasado estrepitosamente.

\footnotetext{
${ }^{15}$ La no contabilización como deuda externa, se debe a que las entidades que administran las represas son "entidades binacionales" autónomas, blindadas con un carácter casi "supranacional".
} 
Los recursos obtenidos de los principales organismos multilaterales prestamistas, no fueron utilizados correctamente. Por tanto, los proyectos ejecutados -sean de infraestructura o de tipos sociales- no están cumpliendo sus fines y objetivos, y de esta manera, no pueden generar los recursos para el repago de los endeudamientos. Pero, el servicio de la deuda hay que pagarlo puntualmente.

\section{Alternativas de solución}

El objetivo fundamental de nuestro estudio ha sido la búsqueda y presentación de nuevos modelos alternativos de financiación para los cuatro sectores analizados.

De acuerdo a nuestro análisis, la estimación de las necesidades financieras para que los problemas más graves de los cuatro sectores puedan solucionarse en un plazo medio de 15 años, ascienden a 7.408.197.382 \$, conforme a lo siguiente:

\begin{tabular}{|c|c|c|}
\hline \multicolumn{3}{|c|}{$\begin{array}{c}\text { Tabla № 9. Estimación de las necesidades financieras prioritarias } \\
\text { para los cuatro sectores analizados }\end{array}$} \\
\hline Sectores & USD & $\%$ \\
\hline Carreteras & 4.535 .202 .625 & 61,2 \\
\hline Salud y saneamiento & 735.677 .775 & 9,9 \\
\hline Educación & 945.827 .382 & 12,8 \\
\hline Vivienda & 1.191 .489 .600 & 16,1 \\
\hline Total & 7.408 .197 .382 & 100,0 \\
\hline
\end{tabular}

Las alternativas de fuentes para nuevas financiaciones que hemos identificado y recomendado en detalle, se citan a continuación en términos muy generales.

\section{I. Renegociaciones de la deuda con los organismos prestamistas}

En lo que atañe a los organismos prestamistas, se sugiere la diversificación de las fuentes de obtención de recursos financieros, así como la negociación para mejorar de manera sustancial las condiciones financieras a ser incluidas en los futuros convenios de préstamos.

En este orden de cosas, se debe negociar la condonación parcial de las deudas. Sobre este espinoso asunto, en un primer paso, habría que concentrarse en la 
condonación del importe total correspondiente al Fondo de Inspección y Vigilancia $(\text { F.I.V.) })^{16}$, el cual representa el $1 \%$ directo que los organismos cobran sobre la suma de cada préstamo.

\subsection{Transformaciones profundas de algunas instituciones del sector público}

En lo referente a las transformaciones de entidades del sector público, se recomienda la reconversión de la Dirección General de Vialidad, organismo dependiente del Ministerio de Obras Públicas y Comunicaciones (MOPC), en una empresa mixta, es decir, con capital proveniente del sector público y privado.

Aunque esta propuesta pueda ser mal vista para los intereses del funcionariado y del gremio sindical del MOPC, el modelo ha funcionado -y funciona- con efectividad en otros países. Al respecto, como hemos observado, el rol de la Dirección General de Vialidad, como gestora de la red vial paraguaya, ha sido lamentable. Por tanto, se debe adoptar una medida drástica para cambiar el rumbo de los acontecimientos, porque los meros cambios y rotaciones de recursos humanos no han conducido a nada.

Asimismo, se propone un programa de reingeniería institucional para el Banco Nacional de Ahorro y Préstamo para la Vivienda (BNAPV), brazo financiero del Consejo Nacional para la Vivienda (CONAVI), convirtiéndose en eje de desarrollo para el sector Vivienda, sobre el cual comentamos más abajo, en el numeral 6.4 .

\subsection{Renegociación del Tratado de Itaipú, con Brasil}

Nuestro estudio no recomienda la renegociación con Brasil del Tratado de Itaipú, propiamente dicha, porque entraríamos en un terreno del derecho internacional público, disciplina que no manejamos. Lo que sí se sugiere es la revisión de lo que concierne a la tasa de interés del préstamo que Brasil concedió a Paraguay. Ciertamente es un tema de extrema delicadeza, pero, clave para las finanzas públicas paraguayas. En este sentido, debemos afirmar que las condiciones finan-

\footnotetext{
${ }^{16}$ ¿Por qué reclamar el FIV? Porque si bien, la administración de los proyectos corren por cuenta de las unidades ejecutoras de los países, los organismos multilaterales perciben este cargo por la responsabilidad que asumen en la inspección y vigilancia durante la ejecución de los proyectos. Si los mismos han fracasado, la reclamación debe ser atendida. Así de sencillo.
} 
cieras vigentes que Brasil impuso a Paraguay -obviamente en contubernio con las antiguas autoridades nacionales paraguayas, a través de la empresa "Eletrobrás, Centrales Eléctricas Brasileñas, S.A."- son usurarias y deben ser modificadas. Nuestro citado trabajo propone y demuestra que la reducción gradual de la tasa vigente $-\mathrm{i} 11,5 \%$ anual! - durante 10 años, hasta alcanzar los niveles de mercado, generaría enormes reducciones de pagos de Paraguay. Asimismo, las estimaciones efectuadas muestran que Brasil, en el largo plazo, también se beneficiaría de la operación.

\subsection{El desarrollo del mercado de capitales}

Frente a la escasez de recursos financieros internacionales para el desarrollo del sector vivienda, esta propuesta ha sido diseñada como alternativa de solución para dicho sector económico. Cabe destacar que la participación y la conjunción de esfuerzos entre el sector privado y público de Paraguay resulta perentoria para dinamizar el mercado bursátil, y a través del mismo, obtener recursos financieros internos y externos para incentivar la construcción de viviendas y paliar el acuciante déficit habitacional ${ }^{17}$.

Si llega a funcionar el modelo propuesto en el mercado financiero nacional, con posterioridad, podría negociarse la participación y el apoyo a esta iniciativa de los mercados bursátiles de San Pablo, Brasil y Buenos Aires, Argentina (BOVESPA y MERVAL, respectivamente). Este apoyo constituiría una muestra importante del ingrediente de solidaridad en la región, término muy utilizado en los discursos políticos de la región, pero, poco aplicado en los hechos del día a día.

En este sentido, si bien el desarrollo y las estrategias políticas tendentes para su logro debe ser un interés genuinamente paraguayo, el ingrediente de la solidari-

\footnotetext{
17 La crisis inmobiliaria surgida en EE.UU., durante el pasado verano, que supuestamente sería la principal causa de distorsión de los mercados financieros mundiales, a primera vista, podría debilitar la alternativa de solución propuesta para el sector vivienda.
}

Sin embargo, a nuestro juicio, los securities fallidos en los mercados financieros estadounidenses -originados, a la vez, en concesiones indebidas de créditos "subprimes"- que configuran una porción marginal en relación a las multimillonarias cifras de activos negociados, no pueden ser la causa principal de la desestabilización financiera mundial. Las causas reales tienen que ser otras.

Además, dentro de unos meses, esta crisis será recordada como otras tantas. Por tanto, nuestra propuesta para el sector vivienda consideramos que sigue siendo enteramente válida. 
dad financiera internacional, muy especialmente de los grandes vecinos, Brasil y Argentina, configura un elemento estratégico para revertir la delicada situación socioeconómica por la que se halla atravesando Paraguay.

\section{Conclusión}

Durante el año 2007, con gran satisfacción, hemos venido leyendo en los periódicos de Paraguay la inquietud generalizada sobre algunas de las alternativas propuestas. A modo de ejemplo, podemos referirnos a la notoriedad pública que ha cobrado el interés por la renegociación del Tratado de Itaipú. Las voces de protesta por este delicado e injusto asunto han cobrado fuerza inusitada durante el año recientemente finalizado.

En este sentido, mencionamos que las elecciones presidenciales en Paraguay se realizarán el 20 de abril de 2008. Y el candidato más fuerte de la oposición, Fernando Lugo ${ }^{18}$, líder del movimiento Alianza Patriótica para el Cambio, incluye la renegociación del Tratado de Itaipú como principal propuesta de su movimiento. Fernando Lugo encabeza claramente los resultados de las últimas encuestas.

Asimismo, en relación a otras alternativas planteadas, hemos podido cerciorarnos de la creciente preocupación de todas las opciones políticas por encarar la planificación de asuntos vitales tales como: la solución de la escasa red vial, las altas tasas de mortalidad infantil y materna, y las agudas precariedades en materia educacional.

Todos estos problemas configuran temas cotidianos, hoy más que nunca, en la prensa paraguaya, seguramente como resultado de la delicada situación socioeconómica que vive el país, que empuja a emigrar a decenas de miles de jóvenes. Nuestra reflexión es siempre la misma: el modelo de financiación para el desarrollo en $\mathrm{Pa}$ raguay, durante la segunda mitad del siglo XX, lamentablemente, ha fracasado.

\footnotetext{
${ }^{18}$ Fernando Lugo es un conocido obispo de la Iglesia Católica. Desde su cargo episcopal se ha involucrado directamente por la causa de los más pobres. De esta manera -frente al descrédito de gran parte de la clase política nacional- a petición de líderes políticos de partidos opositores, así como de sectores empresariales y sindicales, hace dos años renunció a su alto cargo eclesiástico para adentrarse en la oscura y movediza arena política paraguaya. Y, a juzgar por los resultados de las últimas encuestas relacionadas con las próximas elecciones generales, este hecho está siendo sumamente valorado por la mayoría de los paraguayos.
} 